\title{
Resonant and coherent transport through Aharonov-Bohm interferometers with coupled quantum dots
}

\author{
V. Moldoveanu, ${ }^{1,2}$ M. Tolea, ${ }^{1}$ A. Aldea, ${ }^{1}$ and B. Tanatar $^{3}$ \\ ${ }^{1}$ National Institute of Materials Physics, P. O. Box MG-7, Bucharest-Magurele, Romania \\ ${ }^{2}$ Centre de Physique Théorique-CNRS, Case 907 Luminy, 13288 Marseille Cedex 9, France \\ ${ }^{3}$ Department of Physics, Bilkent University, Bilkent, 06800 Ankara, Turkey
}

(Received 21 May 2004; revised manuscript received 13 October 2004; published 31 March 2005)

\begin{abstract}
A detailed description of the tunneling processes within Aharonov-Bohm (AB) rings containing twodimensional quantum dots is presented. We show that the electronic propagation through the interferometer is controlled by the spectral properties of the embedded dots and by their coupling with the ring. The transmittance of the interferometer is computed by the Landauer-Büttiker formula. Numerical results are presented for an $\mathrm{AB}$ interferometer containing two coupled dots. The charging diagrams for a double-dot interferometer and the Aharonov-Bohm oscillations are obtained, in agreement with the recent experimental results of Holleitner et al. [Phys. Rev. Lett. 87, 256802 (2001)] We identify conditions in which the system shows Fano line shapes. The direction of the asymetric tail depends on the capacitive coupling and on the magnetic field. We discuss our results in connection with the experiments of Kobayashi et al. [Phys. Rev. Lett. 88, 256806 (2002)] in the case of a single dot.
\end{abstract}

DOI: 10.1103/PhysRevB.71.125338

PACS number(s): 73.23.Hk, 85.35.Ds, 85.35.Be, 73.21.La

\section{INTRODUCTION}

The electronic transport through Aharonov-Bohm rings with embedded quantum dots (QD's) is a new subject in mesoscopic physics whose complexity competes with the already "classical" problem of persistent currents in closed loops.

Inserting one dot in a ring Yacoby et al. ${ }^{1}$ studied for the first time the transport properties of such systems. The observed Aharonov-Bohm (AB) oscillations of the source-drain signal as the magnetic field is varied proved that the tunneling current through the dot is partially coherent. The experiment presented a striking behavior of the transmittance phase as a function of the gate voltage applied on the dot, at each transmittance peak the phase jumps by $\pi$. Due to the twolead geometry used in this experiment the conductance obeys the Onsager relations and as shown in Ref. 2, this imposes a rigidity of the transmittance phase ( 0 or $\pi$ ). Later on Shuster et al. ${ }^{3,4}$ employed a many lead geometry, the phase evolution being obtained as well as the expected Aharonov-Bohm oscillations. The experimental geometry was generalized by Holleitner et al. ${ }^{5}$ who measured the current through a double-dot $\mathrm{AB}$ interferometer (one QD in each arm of the ring). The main achievement of their setup is that the dots can be coherently coupled and hence the transport becomes more complex. They have also found $\mathrm{AB}$ oscillations of the current and emphasized the formation of coherent molecular states in the two dots. Finally, a recent experiment ${ }^{6}$ with a two-dot $\mathrm{AB}$ ring was performed in a four-lead geometry, the measured transmittance showing peaks in several regimes of the capacitive coupling of the ring. Notably, the phase of the transmittance presents the same increment with $\pi$ when one dot is set to resonance and the capacitive coupling of the second dot is varied around a peak.

A closely related problem is the Fano feature of $\mathrm{AB}$ interferometers. As reported in Ref. 7 a one dot AB interferom- eter shows asymmetric line shapes for the transmittance as a function of the plunger gate voltage, the typical proof of the Fano effect, ${ }^{8-10}$ namely the interference between states belonging to continuous and discrete spectra.

The transport properties of $\mathrm{AB}$ interferometers containing QD's were theoretically studied by two techniques, the scattering approach and the Keldysh formalism in the tight binding picture. The scattering theory was successfully used in Refs. 11-13 to describe the physics of one-dot interferometers, including specific properties of the transmittance phase. The $S$ matrix of the scattering problem is computed by writing the Born expansion for the $T$-operator, the conductance being thereafter obtained via the Landauer-Büttiker formula.

In the tunneling picture the net current from one lead to another is computed by perturbation theory and nonequilibrium Green function techniques. Within this approach one can discuss in detail the co-tunneling spin-dependent processes and finite bias transport. ${ }^{14}$ As discussed recently by Kubala and König ${ }^{15}$ both approaches are equivalent, in spite of the differences between the Hamiltonians (in the tunneling picture the coupling between the ring and dots does not appear explicitly).

The way in which the experiments with $A B$ interferometers can indeed provide the transmittance phase is a subtle point that involves the explicit geometry of the leads used to break the unitarity of the two-lead system. ${ }^{16-19}$

In the present work we study systematically the tunneling and coherence properties of $\mathrm{AB}$ interferometers with QD's, particular attention being payed to the geometry used in the experiments of Holleitner et al. ${ }^{5}$ The idea behind the calculations presented below is the following. The transmittance of the interferometer as a whole is first related to its Green function, by the Landauer-Büttiker formula. Second, it is shown that this Green function can be expressed in terms of two Green functions that describe separately the ring and the 
dots system in the presence of the leads. The lead-ring, leaddots, and ring-dot couplings appear as non-Hermitian selfenergies of an effective Hamiltonian. The latter is obtained by the Feschbach formula ${ }^{20,21}$ which is a useful tool when dealing with Hamiltonians of coupled subsystems. We point out that this step is necessary in order to obtain detailed information about the complex processes within the interferometer. The resonant transport through the device is discussed in connection with the spectral properties of the dots system embedded in the interferometer. Our approach shows clearly that the important role in the resonant transport processes is played by the dots inserted in the ring, the latter providing in turn the suitable geometry for quantum coherence.

We do not consider in this paper the Coulomb repulsion because interaction effects on the transport properties of single and coupled dots were studied extensively in the previous papers ${ }^{22-24}$ and all the analysis presented there remains valid here. The Coulomb interaction can be however easily included in our formalism in the Hartree approximation and the charging effects are satisfactorily described by this approach (see Ref. 11 for a similar discussion of the interaction effects in a one-particle approximation). The main topics we consider in this work are the tunneling and coherence properties of $\mathrm{AB}$ interferometers. The Kondo-type effects which are a subject in itself are not discussed here.

The formalism is presented in Sec. II. Numerical results are discussed in Sec. III in connection with the experimental findings, a qualitative agreement being found. Since we have considered two-dimensional quantum dots the magnetic field dependence of the eigenvalues of the coupled dots system is no longer negligible as in the case of a dot modeled by a single site. It will turn out that the drift of the levels in magnetic field affects the interferometer transport properties. Moreover, the interferometer regime of the device (namely the one that exhibits $\mathrm{AB}$ oscillations) is more difficult to reveal. Section IV summarizes the main results and ends the paper.

\section{FORMALISM}

This section contains the theoretical framework we use to study the electronic transport in Aharonov-Bohm interferometers with coupled quantum dots. The Hamiltonians are written in the tight-binding (TB) representation which is particularly useful both for describing complex geometries and performing numerical computations. We consider a general interferometer that consists of an arbitrary number of twodimensional (coupled) quantum dots embedded in a 1D mesoscopic ring having $N$ sites. Some of these sites are shared with the dots, which are coupled to each other by tunneling Hamiltonians simulating the tunable barriers patterned in experiment. The quantum dots are described as finite twodimensional (2D) plaquettes.

The electrons reach and leave the interferometer through ideal one-channel semi-infinite leads attached on the ring or directly on the dots. The Hamiltonian of the whole system has the form

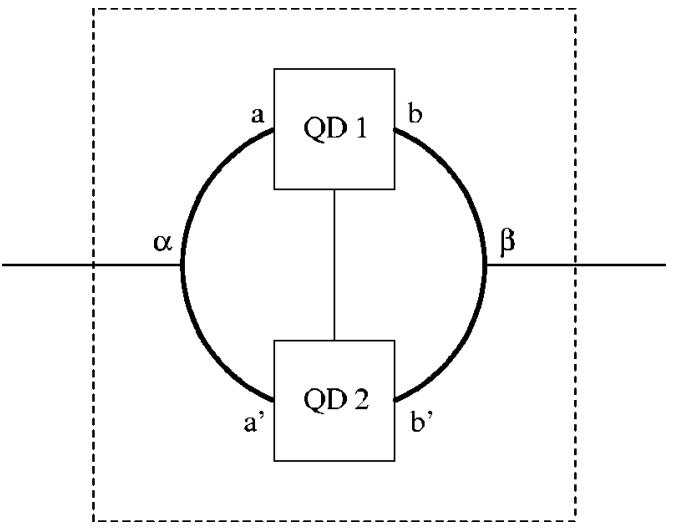

FIG. 1. Schematic picture of a two-dots Aharonov-Bohm interferometer. The thick solid line represents the truncated ring (R). The dashed contour surrounds the interferometer (I). $\alpha, \beta$ are the sites where the leads are connected to the interferometer and $a, a^{\prime}, b, b^{\prime}$ are the contact points between ring and dots.

$$
H=H^{I}+H^{L}+H_{\text {tun }}^{L I}
$$

with

$$
H^{I}=H^{D}+H^{R}+H_{\mathrm{tun}}^{R D} .
$$

$H^{I}$ is the Hamiltonian of the interferometer, $H^{L}$ and $H^{R}$ describe the leads and the truncated ring, i.e., what is left from it after removing the dots (the notations can be identified as well from Fig. 1 which represents a double-dot interferometer). The magnetic flux through the ring appears in $H^{R}$ in the Peierls representation as magnetic phases attached to the hopping constants along the truncated ring. Their explicit form is obtained by using for example the Landau gauge. $H_{\text {tun }}^{L I}$ and $H_{\text {tun }}^{R D}$ are the lead-interferometer and ring-dots tunneling Hamiltonians,

$$
H_{\mathrm{tun}}^{L I}=H^{L I}+H^{I L}=\tau_{L} \sum_{\alpha}\left(\left|0_{\alpha}\right\rangle\langle\alpha|+| \alpha\rangle\left\langle 0_{\alpha}\right|\right)
$$

$$
H_{\mathrm{tun}}^{R D}=H^{D R}+H^{R D}=\tau \sum_{m}\left(e^{-i \varphi_{m}}|m\rangle\left\langle 0 m\left|+e^{i \varphi_{m}}\right| 0 m\right\rangle\langle m|\right) .
$$

Here $\tau_{L}, \tau$ are the corresponding hopping parameters and $0 \alpha(0 m)$ are the nearest sites to the contact points $\alpha(m)$ between lead-interferometer and ring-dots.

$\varphi_{m}$ is the Peierls phase associated with the pair of sites $|0 m\rangle,|m\rangle$. Finally $H^{D}$ is the Hamiltonian of the coupled dots which is also written in the Peierls representation. It includes the individual Hamiltonian of each dot $H^{D_{k}}$,

$$
H^{D_{k}}=-e V_{k} \sum_{i \in Q D_{k}}|i\rangle\left\langle i\left|+t_{D} \sum_{\left\langle i, i^{\prime}\right\rangle} e^{2 \pi i \varphi_{i i^{\prime}}}\right| i\right\rangle\left\langle i^{\prime}\right|
$$

and the interdot tunneling term $H_{\text {tun }}\left(\tau_{\text {int }}\right)$, depending on the coupling constant $\tau_{\text {int }}$ which is the same for each pair of dots $\{k, k+1\}$. We point out that the dots embedded in different arms of the ring can be coupled as well, allowing thus complicated electronic trajectories within the system. The constant term $V_{k}$ from the diagonal part of each $H^{D_{k}}$ mimics the 
plunger gate voltages used in experiments to tune the dots to resonance, $\left\langle i, i^{\prime}\right\rangle$ denotes the nearest neighbor summation and $t_{D}$ is the hopping integral on dots.

The conductance matrix $g_{\alpha \beta}$ of a mesoscopic system at zero temperature coupled to leads is given by the LandauerBüttiker formula (see Ref. 25 for a rigorous derivation)

$$
g_{\alpha \beta}\left(E_{F}\right)=\frac{e^{2}}{h} T_{\alpha \beta}\left(E_{F}\right)=4 \frac{e^{2}}{h} \frac{\tau_{L}^{4}}{t_{L}^{2}} \sin ^{2} k\left|\left\langle\alpha\left|G_{\text {eff }}\left(E_{F}+i 0\right)\right| \beta\right\rangle\right|^{2},
$$

$$
\alpha \neq \beta,
$$

where $T_{\alpha \beta}\left(E_{F}\right)$ is the transmittance, $|\alpha\rangle,|\beta\rangle$ are sites located on the ring or dots that are coupled to the leads, $E_{F}=2 t_{L} \cos k$ is the Fermi energy of the leads and $t_{L}$ is the hopping integral on leads. The main quantity in Eq. (6) is the effective resolvent of the system in the presence of the leads (see Ref. 22 for more details). In our case $G_{\text {eff }}(z)=\left[H_{\text {eff }}(z)\right.$ $-z]^{-1}$, where the effective Hamiltonian is defined as

$$
H_{\text {eff }}(z):=H^{I}-\tau_{L}^{2} \zeta_{1}(z)\left(\sum_{\alpha_{r}}\left|\alpha_{r}\right\rangle\left\langle\alpha_{r}\left|+\sum_{\alpha_{d}}\right| \alpha_{d}\right\rangle\left\langle\alpha_{d}\right|\right)
$$

and acts in the Hilbert space of the interferometer $\mathcal{H}_{I}$ only and embodies the influence of the leads at the contact points with the ring which we denote $\left\{\alpha_{r}\right\}$ or the dots $\left\{\alpha_{d}\right\}$ through the non-Hermitian terms above (these terms represent the so-called leads' self-energy, see Ref. 26). The notation $\zeta_{1}(z)=\left(z \mp \sqrt{z^{2}-4 t_{L}^{2}}\right) / 2[\mp$ shows that $z$ belongs to the upper (lower) half-plane] and we choose $\operatorname{Re} z<2 t_{L}$. In the sequel we take for simplicity $e=h=t_{L}=1$.

In the previous papers ${ }^{22,24}$ we used simpler effective Hamiltonians. In the particular case of a single dot weakly coupled to leads (see Ref. 22) Eq. (6) gives at once the transmittance peaks (as a function of the plunger gate voltage $V$ ) which are related to spectral properties of the dot. Actually the effective Hamiltonian of the dot has resonances with small imaginary part located near the eigenvalues of the isolated dot. Similarly, if $H_{\text {eff }}(z)$ describes an array of identical dots one can obtain and explain the splitting of the Coulomb peaks as a function of the interdot coupling $\tau_{\text {int }}$ in terms of the nearly identical spectra of the dots. Moreover, if the interdot Coulomb interaction is neglected, the effective Green function can be expressed only in terms of one dot Green function by a recursive formula.

Here formula (6) is not of much use because even if the transmittance peaks can be obtained from it by inverting numerically the finite matrix of the effective resolvent, one cannot distinguish between the different paths that an electron can follow. Indeed, due to the ring geometry and to the coupling between the dots the transport within the device is very complex. Besides that, in the experiments the metalic gates defining the dots are patterned in the ring arms while the incident electrons from leads enter the ring freely. This means that $\tau_{L} \sim 1$, thus a discussion in terms of the resonances of $H^{I}$ is useless. These drawbacks are only apparent and one can rewrite $G_{\text {eff }}$ in a suitable way to recover the missing details. The first step is to decompose the Hilbert space of the interferometer as $\mathcal{H}_{I}=\mathcal{H}_{D} \oplus \mathcal{H}_{R}$ where $\mathcal{H}_{D}\left(\mathcal{H}_{R}\right)$ is the Hilbert space of dots (ring). We denote then by $P, Q$ the projectors on these spaces. $P, Q$ are nothing else but families of on-site projections $|i\rangle\langle i|$ from the coupled dots system and the ring. Next, observe that $H_{\text {tun }}^{R D}$ is a small off-diagonal perturbation with respect to $H^{D}-\tau_{L}^{2} \zeta_{1}(z) \Sigma_{\alpha_{d}}\left|\alpha_{d}\right\rangle\left\langle\alpha_{d}\right|$ and $H^{R}-\tau_{L}^{2} \zeta_{1}(z) \Sigma_{\alpha_{r}}\left|\alpha_{r}\right\rangle\left\langle\alpha_{r}\right|$ viewed as non-Hermitian operators in $\mathcal{H}_{D}$ and $\mathcal{H}_{R}$. This allows us to use the Feschbach formula ${ }^{20,21}$ which expresses the effective resolvent in the following form [see Eq. (6.1) from Sec. VI B in Ref. 21]:

$$
\begin{aligned}
G_{\mathrm{eff}}(z)= & G_{\mathrm{eff}}^{R}(z)+\left[1-G_{\mathrm{eff}}^{R}(z) Q H_{\mathrm{eff}}(z) P\right]\left[H_{\mathrm{eff}}^{D}(z)-z\right]^{-1} \\
& \times\left[1-P H_{\mathrm{eff}}(z) Q G_{\mathrm{eff}}^{R}(z)\right],
\end{aligned}
$$

where we denoted $G_{\text {eff }}^{R}(z):=\left[Q H_{\text {eff }}(z) Q-z\right]^{-1}$ and the new effective Hamiltonian reads

$$
H_{\mathrm{eff}}^{D}(z):=P H_{\mathrm{eff}}(z) P-P H_{\mathrm{eff}} Q\left[Q H_{\mathrm{eff}}(z) Q-z\right]^{-1} Q H_{\mathrm{eff}}(z) P .
$$

Noticing that in our case $P H_{\text {eff }}(z) Q=H^{D R}$ one obtains by straightforward calculations explicit formulas for $G_{\text {eff }}^{R}(z)$ and $H_{\text {eff }}^{D}(z)$ [we use the notation $\left.G_{i j}(z):=\langle i|G(z)| j\rangle\right]$,

$$
\begin{aligned}
G_{\mathrm{eff}}^{R}(z):= & \left(H^{R}-\tau_{L}^{2} \zeta_{1}(z) \sum_{\alpha_{r}}\left|\alpha_{r}\right\rangle\left\langle\alpha_{r}\right|-z\right)^{-1}, \\
H_{\mathrm{eff}}^{D}(z):= & H^{D}-\tau_{L}^{2} \zeta_{1}(z) \sum_{\alpha_{d}}\left|\alpha_{d}\right\rangle\left\langle\alpha_{d}\right| \\
& -\tau^{2} \sum_{m, m^{\prime}} e^{-i\left(\varphi_{m^{-}}-\varphi_{m^{\prime}}\right)} G_{0 m, 0 m^{\prime}}^{R}(z)|m\rangle\left\langle m^{\prime}\right| .
\end{aligned}
$$

The advantage of using the Feschbach formula is that it provides us with two effective resolvents, each one describing individually the pieces that compose the interferometer. $G_{\text {eff }}^{R}$ describes the truncated ring in the presence of the leads while $G_{\text {eff }}^{D}(z):=\left(H_{\text {eff }}^{D}-z\right)^{-1}$ is an effective resolvent for the embedded system of dots both in the presence of leads and ring. We remark that $G_{0 m, 0 m^{\prime}}^{R}(z)$ [see Eq. (10)] has a nonvanishing imaginary part even if $z$ lies on the real axis, due to the non-Hermitian coupling to the leads. This happens because $\zeta_{1}(E)$ is always complex when $|E|<2 t_{L}$. By direct computation we express various elements of the conductance matrix using Eq. (8) (this time the $E_{F}$ dependence is omitted as well as the subscript "eff.")

$$
\begin{gathered}
g_{\alpha_{r}, \beta_{r}}=4 \tau_{L}^{4} \sin ^{2} k \mid G_{\alpha_{r}, \beta_{r}}^{R} \\
+\left.\tau^{2} e^{i\left(\varphi_{m}-\varphi_{n}\right)} G_{\alpha_{r}, 0 m}^{R} G_{m n}^{D} G_{0 n, \beta_{r}}^{R}\right|^{2}=:\left|t_{\alpha_{r}, \beta_{r}}^{R}+t_{\alpha_{r}, \beta_{r}}^{Q D}\right|^{2}, \\
g_{\alpha_{r}, \beta_{d}}=4 \tau_{L}^{4} \tau^{2} \sin ^{2} k\left|G_{\alpha_{r}, 0 m}^{R} G_{m, \beta_{d}}^{D}\right|^{2}, \\
g_{\alpha_{d}, \beta_{d}}=4 \tau_{L}^{4} \sin ^{2} k\left|G_{\alpha_{d}, \beta_{d}}^{D}\right|^{2} .
\end{gathered}
$$

In the above equations the summations over $m$ and $n$ are understood. The set of formulas (12)-(14) is the main formal result of the paper and the starting point of a detailed discussion of the transport processes through the system in terms of 


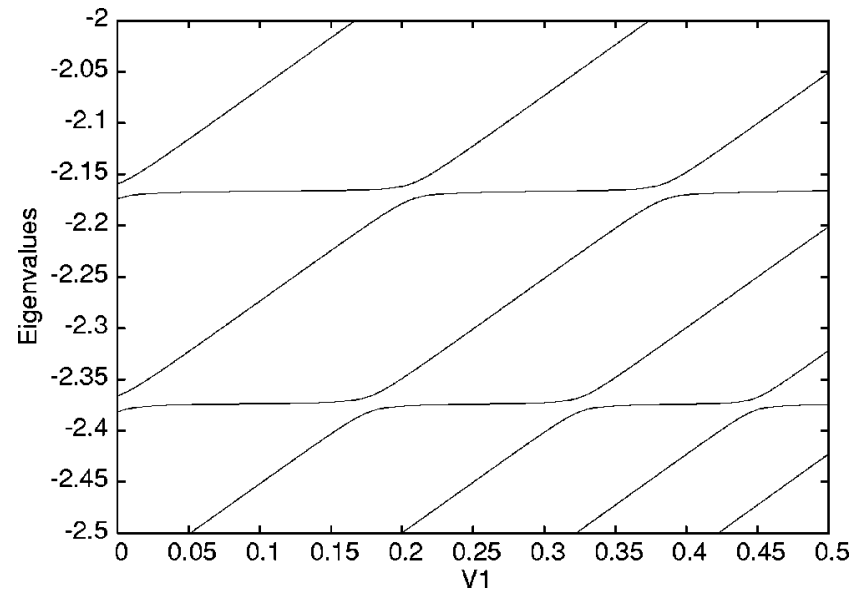

FIG. 2. Avoided crossings in the spectrum of a $20 \times 10$ double dot as a function of the detuning potential $V_{1}$ applied on $\mathrm{QD}_{1}\left(\tau=0.4, \Phi=0.15, \tau_{\text {int }}=0.1\right)$. Here $\Phi$ is the magnetic flux through one cell, in flux quanta.

the spectral properties of the effective Hamiltonian $H_{\mathrm{eff}}^{D} \cdot t_{\alpha_{r}, \beta_{r}}^{R}$ is the transmission amplitude from lead $\alpha$ to lead $\beta$ via the truncated ring and $t_{\alpha_{r}, \beta_{r}}^{Q D}$ controls the transport via the arm containing the $\operatorname{dot}(\mathrm{s})$. In the following we consider some particular geometries already used in experiments.

\section{A. One-dot $A B$ interferometer}

The simplest $\mathrm{AB}$ device is realized when there are no leads attached to the dots system which in turn is composed of only one dot (this is the geometry used by Yacoby et al. ${ }^{1}$ ). Then the term containing the sites $\left\{\alpha_{d}\right\}$ vanishes from Eq. (11) and the transport is completely described by Eq. (12) that gives the transmittance of the system.

Let $E_{i}(V)$ be the $i$ th eigenvalue of the isolated dot, $\psi_{i}$ the corresponding eigenfunction and $P_{i}:=\left|\psi_{i}\right\rangle\left\langle\psi_{i}\right|$ its associated projection. Note that the eigenvalues $E_{i}(V)$ and their eigenfunctions $\left|\psi_{i}\right\rangle$ depend also parametrically on the magnetic field. We describe below the resonant transport through $E_{i}(V)$. The idea is to isolate the resonant contribution in the effective resolvent. With the notation $P_{i}^{\perp}:=1-P_{i}$, the effective Hamiltonian can be written as

$$
H_{\mathrm{eff}}^{D}=P_{i} H_{\mathrm{eff}}^{D} P_{i}+P_{i} H_{\mathrm{eff}}^{D} P_{i}^{\perp}+P_{i}^{\perp} H_{\mathrm{eff}}^{D} P_{i}+P_{i}^{\perp} H_{\mathrm{eff}}^{D} P_{i}^{\perp},
$$

and we can apply again the Feschbach lemma for $\left(H_{\text {eff }}^{D}-z\right)^{-1}$ having $P_{i} H_{\text {eff }}^{D} P_{i}^{\perp}+$ H.c. as a small perturbation of $P_{i} H_{\text {eff }}^{D} P_{i}+P_{i}^{\perp} H_{\text {eff }}^{D} P_{i}^{\perp}$. Then with the notations $G_{i}^{\perp}:=\left(P_{i}^{\perp} H_{\mathrm{eff}}^{D} P_{i}^{\perp}-z\right)^{-1}$ and $D_{i}(z):=P_{i} H_{\mathrm{eff}}^{D} P_{i}^{\perp} G_{i}^{\perp} P_{i}^{\perp} H_{\mathrm{eff}}^{D} P_{i}$ the effective resolvent reads

$$
\begin{aligned}
{\left[H_{\mathrm{eff}}^{D}(z)-z\right]^{-1}=} & G_{i}^{\perp}+\left(1-G_{i}^{\perp} P_{i}^{\perp} H_{\mathrm{eff}}^{D} P_{i}\right)\left(P_{i} H_{\mathrm{eff}}^{D} P_{i}-D_{i}-z\right)^{-1} \\
& \times\left(1-P_{i} H_{\mathrm{eff}}^{D} P_{i}^{\perp} G_{i}^{\perp}\right)
\end{aligned}
$$

and the resonant term is clearly
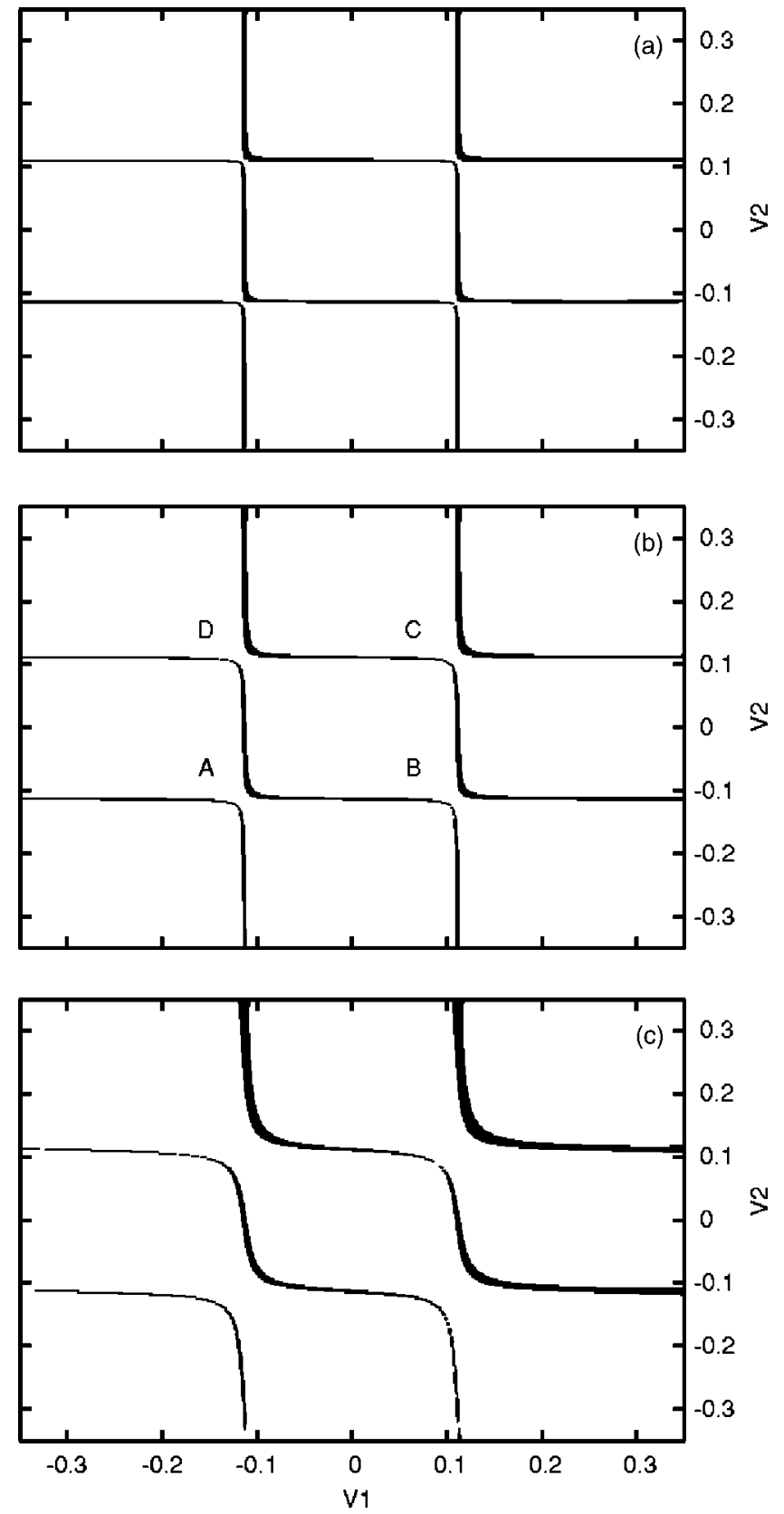

FIG. 3. Charging diagrams for the double-dot Aharonov-Bohm interferometer $\left(\tau_{L}=1, \phi=3, \tau=0.3\right) . \varphi$ is the magnetic flux through the ring, in flux quanta. The traces represent transmittances bigger than 0.4. (a) $\tau_{\text {int }}=0.1$, (b) $\tau_{\text {int }}=0.2$, (c) $\tau_{\text {int }}=0.5$. The Fermi level is set to 0 .

$$
\left[P_{i} H_{\mathrm{eff}}^{D}(z) P_{i}-D_{i}(z)-z\right]^{-1}=\frac{\left|\psi_{i}\right\rangle\left\langle\psi_{i}\right|}{E_{i}(V)-\Delta_{i}(z)-i \Gamma_{i}(z)-z},
$$

where the resonance width $\Gamma_{i}$ and the shift $\Delta_{i}$ are fluxdependent quantities, their expressions being easily identified. Notice also that $\left\langle\psi_{i}\left|D_{i}(z)\right| \psi_{i}\right\rangle$ is of order $\tau^{4}$ thus $\Gamma_{i}(z)$ is of order $\tau^{2}$. Let now $z \rightarrow E_{F}+i 0$ and suppose that we fix $V$ such that $E_{i}(V)=E_{i}-V \simeq E_{F}+\Delta_{i}\left(E_{i}\right.$ being the eigenvalue of the dot in the absence of the capacitive coupling). It is clear 


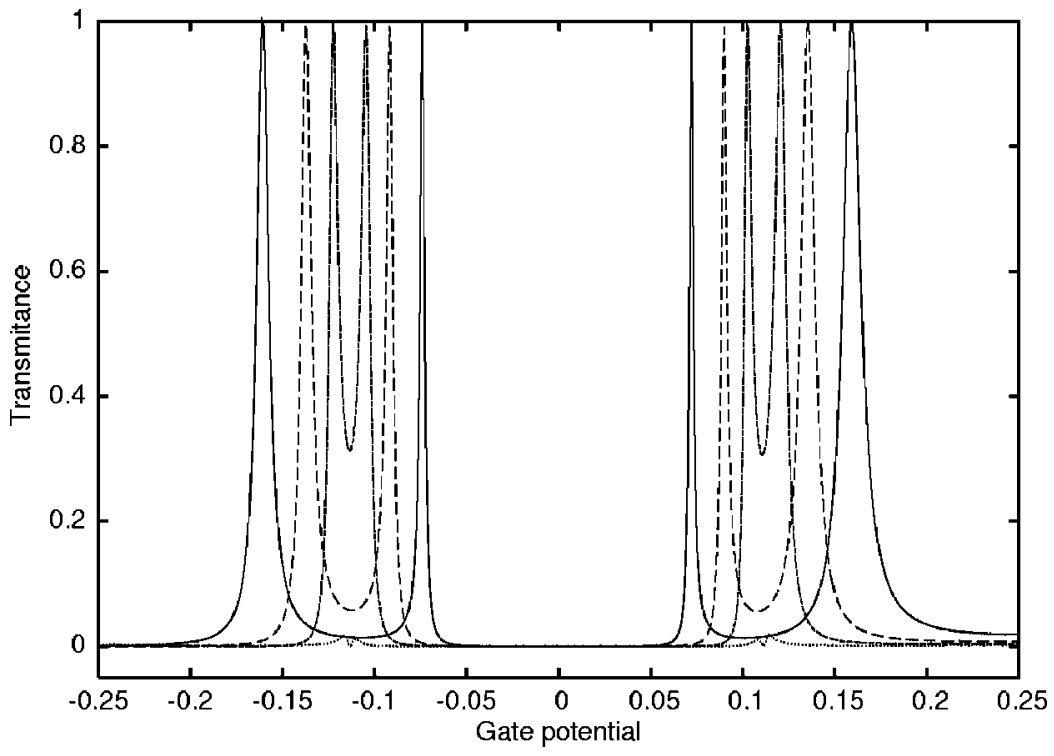

FIG. 4. The effects of the interdot coupling $\tau_{\text {int }}$ on the electronic transmittance of a doubledot Aharonov-Bohm interferometer at fixed magnetic flux $\phi=3$. The same gate potential $V$ is applied on each dot $\tau_{L}=1, \tau=0.5$. Full line, $\tau_{\text {int }}=1$; long dashed line, $\tau_{\text {int }}=0.5$; dashed line, $\tau_{\text {int }}=0.2$; dotted line, $\tau_{\text {int }}=0$ (transport is strongly suppressed),

that the main contribution in (16) comes from $\left[P_{i} H_{\mathrm{eff}}^{D}(z) P_{i}\right.$ $\left.-D_{i}(z)-z\right]^{-1}$ since $G_{i}^{\perp}$ stays bounded and the other terms are of $\mathcal{O}\left(\tau^{2}\right)$. The denominator of $\left[P_{i} H_{\text {eff }}^{D}(z) P_{i}-D_{i}(z)-z\right]^{-1}$ reduces to resonance width $\Gamma_{i}$ which compensates the multiplicative factor $\tau^{2}$ from the numerator of $t_{\alpha_{r}, \beta_{r}}^{Q D}$. This behavior induces a peak in $t_{\alpha_{r}, \beta_{r}}^{Q D}$ and hence in the total transmittance across the ring. With these considerations we conclude that for weak ring-dot coupling, whenever $V$ comes close to $E_{F}-E_{i}$ for some $E_{i}$ the transmittance can be written in the form

$t_{\alpha_{r}, \beta_{r}}^{Q D}=2 i \tau_{L}^{2} \tau^{2} \sin k G_{\alpha_{r}, 0 m}^{R} G_{0 n, \beta_{r}}^{R} \frac{e^{i\left(\varphi_{m}-\varphi_{n}\right)}\left\langle m \mid \psi_{i}\right\rangle\left\langle\psi_{i} \mid n\right\rangle}{E_{i}-V-\Delta_{i}-E_{F}-i \Gamma_{i}}+\mathcal{O}\left(\tau^{2}\right)$.

Equation (18) is a Breit-Wigner-type formula and gives the transmittance between the leads via the quantum dot, as measured in Refs. 1-3. A similar formula was obtained by Hackenbroich and Weidenmüller for a continuous model. ${ }^{12,13}$ They supposed that $E_{i}$ is flux independent, which is true only at low magnetic fields and small dots. This assumption permits an analytical discussion of the flux-dependence of $t_{\alpha_{r}, \beta_{r}}^{Q D}$. As we have said, here we shall not neglect the effect of the magnetic field on the dot levels. We also point out that the one resonance form for the dot transmittance was obtained here starting from a many-level description of the dot. The rigorous argument for using from the beginning this simplified form is that after subtracting the resonant term from the effective resolvent the remainder is nonsingular and small.

\section{B. AB interferometer with a coherent double dot}

When $H^{D}$ describes two coupled dots embedded in different arms of the ring connected to two leads (see Fig. 1) we recover the setup of Holleitner et al. ${ }^{5}$ In the absence of the lead-dot coupling Eqs. (13) and (14) give no contribution thus we are left only with Eq. (12). For the simplicity of writing we shall denote $G_{\alpha_{r}, 0 m}^{R}:=G_{\alpha_{r}, m}^{R}$ and $\varphi_{m}-\varphi_{m^{\prime}}:=\theta_{m m^{\prime}}$. Since $G_{\alpha \beta}^{R}=0$ in this case the conductance has the form

$$
\begin{aligned}
g_{\alpha \beta}= & 4 \tau_{L}^{4} \tau^{4} \sin ^{2} k \mid e^{i \theta_{a b}} G_{\alpha a}^{R} G_{a b}^{D} G_{b \beta}^{R}+e^{i \theta_{a^{\prime} b^{\prime}}} G_{\alpha a^{\prime}}^{R} G_{a^{\prime} b^{\prime}}^{D} G_{b^{\prime} \beta}^{R} \\
& +e^{i \theta_{a b^{\prime}}} G_{\alpha a}^{R} G_{a b^{\prime}}^{D} G_{b^{\prime} \beta}^{R}+\left.e^{i \theta_{a^{\prime} b}} G_{\alpha a^{\prime}}^{R} G_{a^{\prime} b}^{D} G_{b \beta}^{R}\right|^{2} .
\end{aligned}
$$

We remark that the terms $G_{a^{\prime} b}^{D}$ and $G_{a b^{\prime}}^{D}$ connect points that belong to different dots. The effective Hamiltonian in this case is

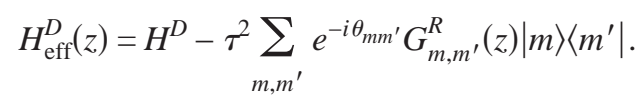

As in the preceding section, we are interested in discussing the resonant transport in terms of the spectral properties of the coupled dots system. Since the double-dot Hamiltonian $H^{D}$ depends parametrically on the capacitive couplings $V_{1}, V_{2}$ we denote its eigenvalues and eigenfunctions by $E_{i}\left(V_{1}, V_{2}\right)$ and $\psi_{i}\left(V_{1}, V_{2}\right)$. The main point is that for suitable pairs $\left\{V_{1}, V_{2}\right\}$ one can bring $E_{i}\left(V_{1}, V_{2}\right)$ close to $E_{j}\left(V_{1}, V_{2}\right)$ for $j=i+1$. This is due to the spectral properties of detuned dots. Let us remind here that the detuning consists in applying an additional gate potential to one dot while keeping the other gate voltage fixed. In Fig. 2 we show the spectrum of the detuned double dot $(10 \times 10$ sites on each dot $)$ as a function of the detuning potential $V_{1}$ applied on the first dot, for a fixed value of $\tau_{\text {int }}$. For simplicity the undetuned dot is not capacitively coupled thus $V_{2}=0$. Obviously, one-half of the spectrum shifts linearly in $V_{1}$. The remaining eigenvalues depend weakly on $V_{1}$, excepting some points of avoided crossings. As long as $\tau_{\text {int }} \neq 0$ there are no crossings between eigenvalues (on the contrary, as shown in Fig. 2 we rather have avoided crossings). Moreover, by perturbation theory, near avoided crossings the distance between eigenvalues is of order $\tau_{\text {int }}$. This behavior of eigenvalues as functions of $V_{1}$ and $V_{2}$ is due to the fact that, roughly speaking, half of the eigenvalues belong to $\mathrm{QD}_{1}$, the other half to $\mathrm{QD}_{2}$. As a consequence, when $V_{1}, V_{2}$ are tuned such that both $E_{i}\left(V_{1}, V_{2}\right)$ and $E_{j}\left(V_{1}, V_{2}\right)$ are near and moreover close to the Fermi level we expect that both dots will transmit. Clearly one can study the tunneling through one eigenvalue following the same 
steps as in the analysis of a single-dot case. The interesting situation is however the one in which the resonant tunneling involves both eigenvalues. In the following we show how this appears formally at the level of $G^{D}$. To this end let us introduce the two-dimensional projection $P_{i j}:=P_{i}+P_{j}, P_{k}$ being the projection associated to the eigenvalue $E_{k}\left(V_{1}, V_{2}\right)$ with $k=i, j$ for $i$ and $j$ fixed. We shall also use the notation $P_{i j}^{\perp}:=1-P_{i j}$. Then $P_{i j} H_{\text {eff }}^{D} P_{i j}^{\perp}+$ H.c. is a perturbation [of $\left.\mathcal{O}\left(\tau^{2}\right)\right]$ to $P_{i j} H_{\text {eff }}^{D} P_{i j}+P_{i j}^{\perp} H_{\text {eff }}^{D} P_{i j}^{\perp}$ and by the Feschbach lemma for $G_{\text {eff }}^{D}$ one has

$$
G_{\mathrm{eff}}^{D}=\left(P_{i j}^{\perp} H_{\mathrm{eff}}^{D} P_{i j}^{\perp}-z\right)^{-1}+\left[\tilde{H}_{\mathrm{eff}}^{D}(z)-z\right]^{-1}+\mathcal{O}\left(\tau^{4}\right),
$$

with

$$
\begin{aligned}
\widetilde{H}_{\mathrm{eff}}^{D}(z):= & P_{i j} H_{\mathrm{eff}}^{D}(z) P_{i j}-P_{i j} H_{\mathrm{eff}}^{D} P_{i j}^{\perp}\left(P_{i j}^{\perp} H_{\mathrm{eff}}^{D} P_{i j}^{\perp}-z\right)^{-1} \\
& \times P_{i j}^{\perp} H_{\mathrm{eff}}^{D} P_{i j}=: H^{D}(z)-D_{i j}(z) .
\end{aligned}
$$

As in the case of a single dot the first term in (21) is small when $z \rightarrow E_{F}+i 0$ and the gate voltages are chosen such that the resonant condition is fulfilled at least around one of the two eigenvalues $E_{k}\left(V_{1}, V_{2}\right)$. Then the last step to be done is to write the Dyson expansion of $\left(\tilde{H}_{\text {eff }}^{D}-z\right)^{-1}$ taking
$P_{i} \widetilde{H}_{\text {eff }}^{D} P_{j}+$ H.c. $:=V$ as a perturbation of the $2 \times 2$ diagonal matrix $P_{i} \widetilde{H}_{\text {eff }}^{D} P_{i}+P_{j} \widetilde{H}_{\text {eff }}^{D} P_{j}$,

$$
\widetilde{G}_{\mathrm{eff}}^{D}=\widetilde{G}_{i j, \mathrm{eff}}^{D}(z)+\widetilde{G}_{i j, \mathrm{eff}}^{D}(z) V \widetilde{G}_{i j \mathrm{eff}}^{D}+\cdots,
$$

where the unperturbed resolvent $\widetilde{G}_{i j \text {,eff }}^{D}=: G^{(i)}+G^{(j)}$ has the form

$$
\begin{aligned}
\tilde{G}_{i j \text {,ff }}^{D}(z)= & \frac{\left|\psi_{i}\right\rangle\left\langle\psi_{i}\right|}{E_{i}\left(V_{1}, V_{2}\right)-\Delta_{i}(z)-i \Gamma_{i}(z)-z} \\
& +\frac{\left|\psi_{j}\right\rangle\left\langle\psi_{j}\right|}{E_{j}\left(V_{1}, V_{2}\right)-\Delta_{j}(z)-i \Gamma_{j}(z)-z} .
\end{aligned}
$$

The indices $i, j$ were explicitly written for the unperturbed operator (we did not introduce another notation). Thus, we have here two resonances of widths $\Gamma_{i}, \Gamma_{j}$ [their expressions are complicated but easy to obtain from (22)]. It is clear that as long as the dots are coupled $E_{i}\left(V_{1}, V_{2}\right) \neq E_{j}\left(V_{1}, V_{2}\right)$ thus the two resonances come close but do not cross. Indeed, if for some $V_{1}, V_{2}$ the first term in (24) behaves like $1 / \Gamma_{i}$ the denominator of the second term is $\tau_{\text {int }}+\left(\Delta_{i}-\Delta_{j}\right)-i \Gamma_{j}$ thus the resonant condition is not strictly achieved. Let us write explicitly the second term from the Dyson expansion (23). Since $V$ is off-diagonal one is left with

$$
\widetilde{G}_{i j \text {,eff }}^{D}(z) V \widetilde{G}_{i j \text {,eff }}^{D}(z)=\frac{\left|\psi_{i}\right\rangle\left\langle\psi_{j}\left|H_{\text {eff }}^{D}+D_{i j}(z)\right| \psi_{i}\right\rangle\left\langle\psi_{j}\right|+\text { H.c. }}{\left(E_{i}\left(V_{1}, V_{2}\right)-\Delta_{i}(z)-i \Gamma_{i}(z)-z\right) \cdot\left(E_{j}\left(V_{1}, V_{2}\right)-\Delta_{j}(z)-i \Gamma_{j}(z)-z\right)} .
$$

Looking at (20) and (22) one notes that the numerator is quadratic in $\tau$ as well as the widths of the resonances $\Gamma_{i}, \Gamma_{j}$. Thus the perturbative expansion (23) cannot be used in the case of decoupled dots since $E_{i}$ and $E_{j}$ can cross, the imaginary parts of the resonances are equal $\Gamma_{i}=\Gamma_{j}=\Gamma$ and $\tilde{G}_{i j \text {,eff }}^{D} V \widetilde{G}_{i j \text {,eff }}^{D}$ behaves also like $1 / \Gamma$. However here we deal with coupled dots and as long as $\tau_{\text {int }}>\tau^{2}$ the Dyson series (23) converges and (19) becomes (omitting the indexes $i, j$ and eff in $\widetilde{G}_{i j \text {,eff }}^{D}$ )

$$
\begin{aligned}
& g_{\alpha \beta}=4 \tau_{L}^{4} \sin ^{2} k \mid \tau^{2}\left[e^{i \theta_{a b} G_{\alpha a}^{R}} \widetilde{G}_{a b}^{D} G_{b \beta}^{R}+e^{i \theta_{a^{\prime} b^{\prime}} G_{\alpha a^{\prime}}^{R}} \widetilde{G}_{a^{\prime} b^{\prime}}^{D} G_{b^{\prime} \beta}^{R}\right.
\end{aligned}
$$

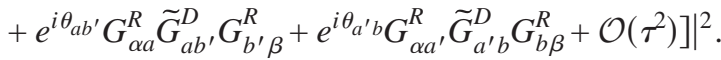

The last formula allows a discussion of the interferometer properties of the device. The first two terms represent the direct tunneling through the upper and lower dot, while the terms containing $\widetilde{G}_{a b^{\prime}}^{D}$ and $\widetilde{G}_{a^{\prime} b}^{D}$ describe paths in which the electron tunnels from one dot to the other before being transmitted in the leads. At small interdot coupling the cross products $\left\langle a \mid \psi_{k}\right\rangle\left\langle\psi_{k} \mid b^{\prime}\right\rangle,\left\langle a^{\prime} \mid \psi_{k}\right\rangle\left\langle\psi_{k} \mid b\right\rangle,\left\langle a \mid \psi_{j}\right\rangle\left\langle\psi_{j} \mid b\right\rangle, \quad$ and $\left\langle a^{\prime} \mid \psi_{i}\right\rangle\left\langle\psi_{i} \mid b^{\prime}\right\rangle$ are expected to be small so that we can write [keeping only the first two terms from the right-hand side of Eq. (26)]

$$
\begin{aligned}
g_{\alpha \beta}= & 4 \tau_{L}^{4} \sin ^{2} k \mid \tau^{2}\left(\frac{e^{i \theta_{a b}} G_{\alpha a}^{R}\left\langle a \mid \psi_{i}\right\rangle\left\langle\psi_{i} \mid b\right\rangle G_{b \beta}^{R}}{E_{i}\left(V_{1}, V_{2}\right)-\Delta_{i}-i \Gamma_{i}-z}\right. \\
& \left.+\frac{e^{i \theta_{a^{\prime} b^{\prime}} G_{\alpha a^{\prime}}^{R}\left\langle a^{\prime} \mid \psi_{j}\right\rangle\left\langle\psi_{j} \mid b^{\prime}\right\rangle G_{b^{\prime} \beta}^{R}}}{E_{j}\left(V_{1}, V_{2}\right)-\Delta_{j}-i \Gamma_{j}-z}\right)+\left.\mathcal{R}\right|^{2},
\end{aligned}
$$

where $\mathcal{R}$ collect all the other paths within the interferometer that give smaller contributions. Equation (27) will help us to discuss the numerical results from the next section.

One may notice that in the above analysis the spectral properties of the truncated ring do not appear in an essential way in the problem. This could be anticipated from the beginning since it is the double-dot system that controls the tunneling events. At the formal level, this fact is revealed only by using the Feschbach formula.

We mention that our Eqs. (12)-(14) and (18) are similar to the ones obtained previously by Hackenbroich and Weidenmüller ${ }^{11,12}$ by a scattering theory approach, in the case of a single dot embedded in a ring connected to two leads. Here we gave an alternative calculation in terms of the Green functions rather than using the $S$ matrix and we generalized the discussion beyond the single-dot case. An advan- 

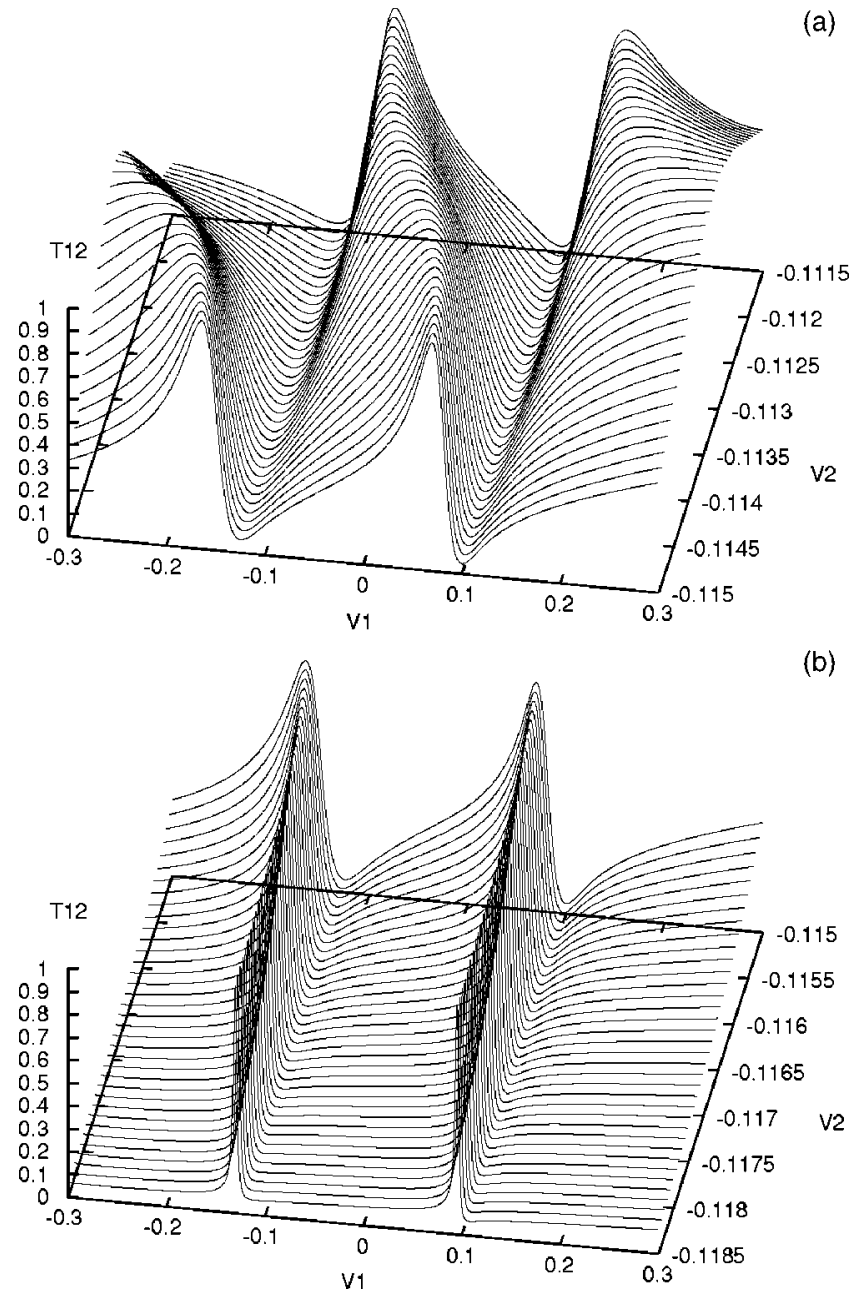

FIG. 5. The structure of the transmittance peaks from Fig. 3(b) around the points of double resonance (a). Away from this point one has distinct sharp peaks that turn into Fano peaks at the avoided crossing points (b).

tage of our approach is that we do not use the Born series which is formally resummed in the scattering approach.

Let us finally observe that one could not compute the tunneling current through the interferometer via rate equation methods used previously in earlier works ${ }^{27,28}$ for weakly coupled quantum dots. These approaches would imply in our problem either the computation of the probability distribution $P(N, \alpha)$ characterizing the interferometer in the $N$-particle state $\alpha$, either a perturbative expansion with respect to the lead-interferometer tunneling Hamiltonian. Since the lead-ring coupling constant is rather big the number of electrons in the interferometer is not quantized, thus $P(N, \alpha)$ is not well-defined, and the perturbative argument breaks down.

\section{RESULTS AND DISCUSSION}

We start this section with the most interesting geometry, the one used by Holleitner et al. ${ }^{5}$ Following their analysis we first look for the charging diagrams of a ring with two identical dots connected to two leads. The dots have $4 \times 5$ sites
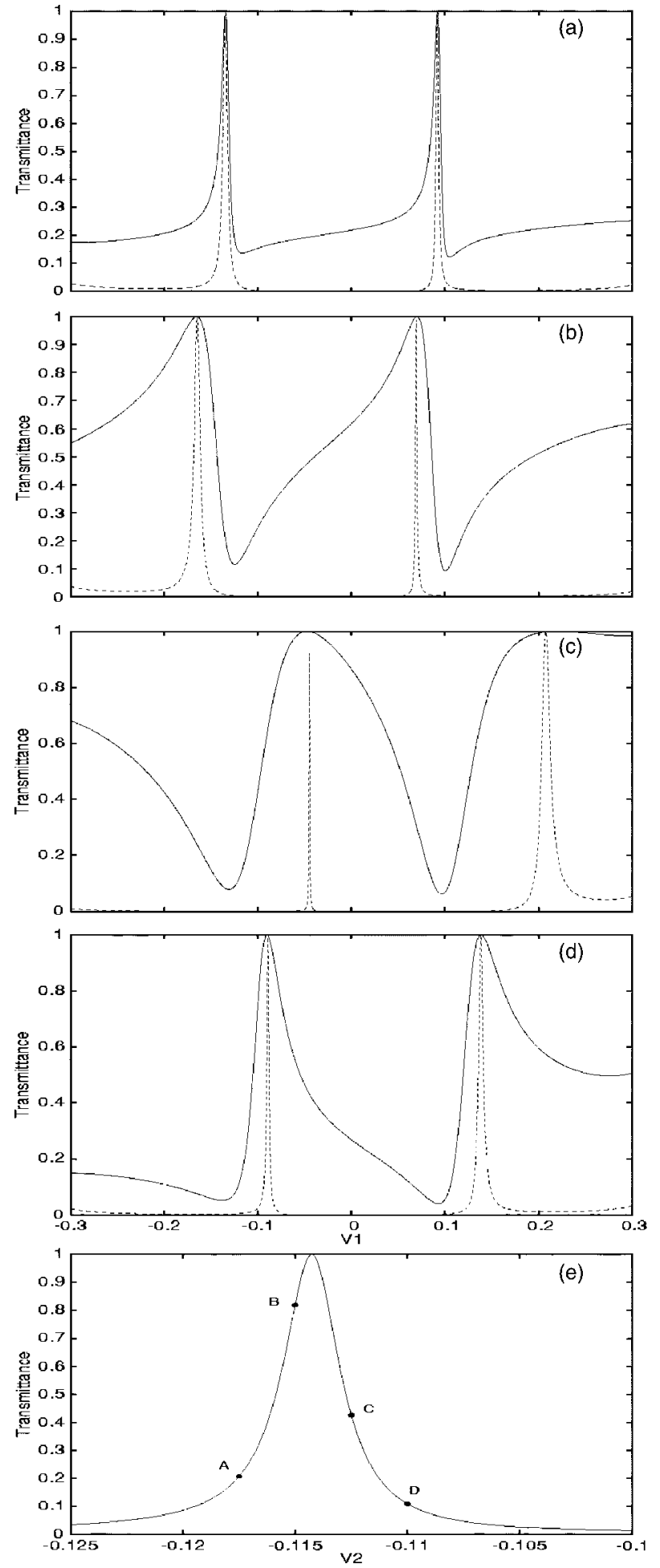

FIG. 6. (a)-(d) Solid lines, Fano line shapes as a function of $V_{1}$ for several values of $V_{2}$. The Fano tail changes its orientation by passing through a symmetric maxima. Dashed lines, the resonant transport through the upper arm of the ring when the lower arm is decoupled from leads. Remark the correspondence between the usual peaks and the Fano maxima. (a) $V_{2}=-0.1175$, (b) $V_{2}=$ -0.1150 , (c) $V_{2}=-0.1125$, (d) $V_{2}=-0.1100$. (e) A resonant peak as a function of $V_{2}$. The gate potential on QD1 was set to $V_{1}=-0.2$. The points $A, B, C, D$ correspond to the values of $V_{2}$ chosen in (a)-(d). All plots are made for $\tau=0.3, \tau_{L}=1, \tau_{\text {int }}=0.2, \phi=3$. 


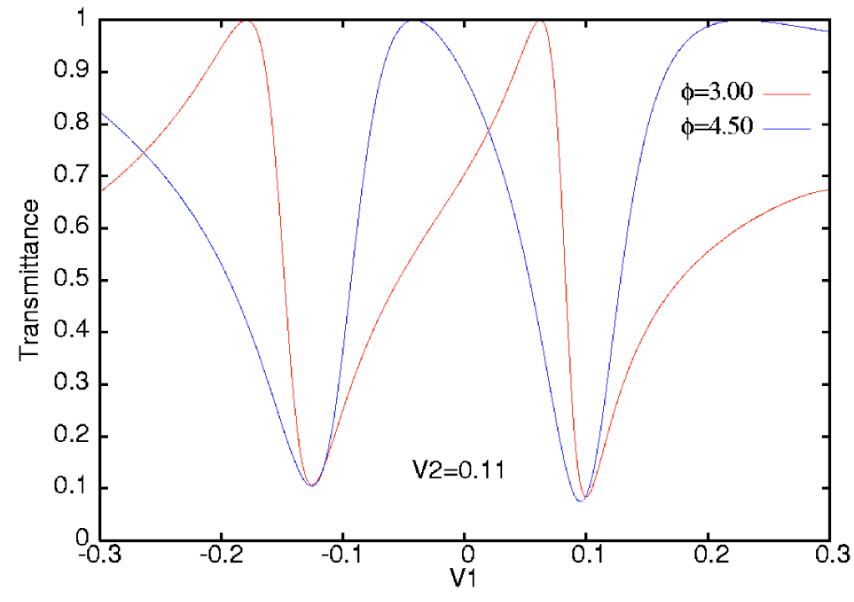

FIG. 7. (Color online) Magnetic control of the Fano interference. As the magnetic field is varied the Fano parameter changes sign. $\tau=0.3, \tau_{L}=1, \tau_{\text {int }}=0.2$.

each, while the ring supports 100 sites. We recall (see also Ref. 29) that the charging diagrams are plots of the current through a system containing two quantum dots as a function of the gate voltages $V_{1}, V_{2}$ applied on each dot. In Fig. 3 we present the rhomboids for our system, obtained as follows: for each fixed value of $V_{2}$, we varied $V_{1}$ in the interval shown in the figures and we selected only transmittances (i.e., conductances) $T_{12}$ that are larger than 0.4 , which means that what we obtain is roughly a map for the peak positions in the plane $\left(V_{1}, V_{2}\right)$. The magnetic flux is fixed. As the interdot coupling increases the diagram changes, due to the usual behavior of the transmittance in coupled dots, ${ }^{30}$ a regular peak is split into two subpeaks, separated by a distance which increases with $\tau_{\text {int }}$ and saturates at perfect coupling $\left(\tau_{\text {int }}=1\right)$. The tunnel split peaks of the interferometer transmittance were observed in Ref. 5 both in vanishing and strong magnetic fields [see Figs. 4(a) and 4(b) in the cited reference]. Figure 4 shows our result for the transmittance of the interferometer at uniform capacitive coupling (i.e., $V_{1}=V_{2}$ ), fixed magnetic flux and different interdot tunneling constants (here $\tau_{r}$ and $\tau$ are also fixed). A striking feature is observed in the case of a ring with decoupled dots (the dotted line in Fig. 4), the transport is strongly suppressed. This behavior at $\tau_{\text {int }}=0$ was predicted also in Ref. 15. It differs from the one encountered in the case of double dots connected directly to leads, when two subpeaks merge to a single one as $\tau_{\text {int }} \rightarrow 0$.

Another important aspect of the charging diagram is the drift of the peaks near double resonance points, which actually gives the honeycomb pattern. We discuss this in connection with Fig. 3(b) using the spectral properties of the detuned dots emphasized in Sec. II B. The traces from the range $V_{2} \in(0.11,0.35)$ depend weakly on $V_{2}$ because the corresponding eigenvalues of the embedded double dot have this behavior there. A similar behavior is observed with the traces in the interval $V_{1} \in(-0.35,-0.11)$ where the eigenvalues depend weakly on $V_{1}$. This behavior changes drastically when two traces are approaching (around point $D$ marked in the figure), they clearly avoid each other, because the eigenvalues of the double dot do not cross $\left(\tau_{\text {int }}=0.2\right)$. The avoided
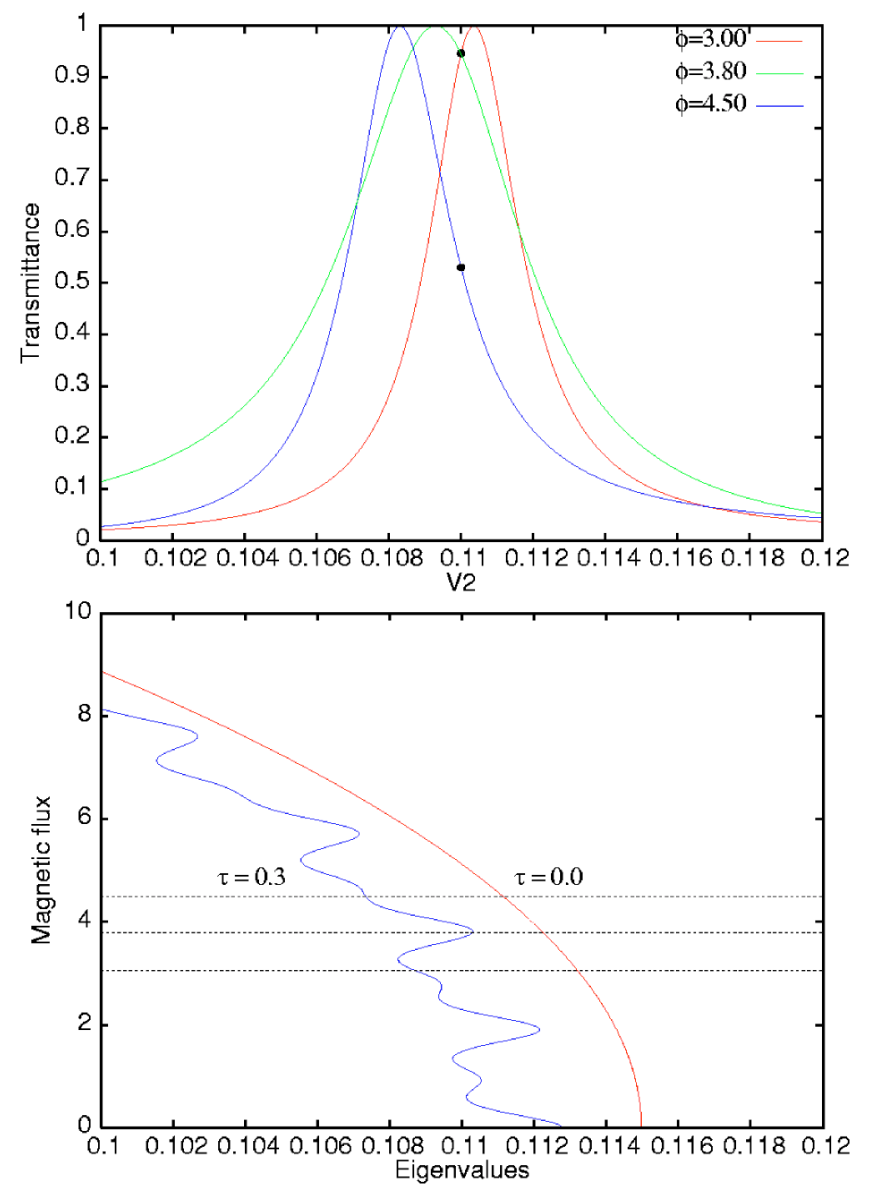

FIG. 8. (Color online) (a) The background peak moves with the magnetic flux; the gate potential on QD1 was set to $V_{1}=-0.2$. (b) The eigenvalue of the decoupled double dot $(\tau=0.0)$ has a positive slope with respect to the magnetic flux. The interferometer eigenvalue $(\tau=0.3)$ is additionally modulated by the hybridization between the truncated ring and the double dot.

crossing is more difficult to discern at small interdot coupling, as in Fig. 3(a). The problem of crossing resonances in double-dot $A B$ interferometers is discussed in a recent work $^{31}$ where it was proved that actually at real energies such crossings do not exist. This result coincides with ours.

In Ref. 5, the interferometer properties of the system were revealed by the following procedure: for a fixed avoided crossing of the charging diagram the current through the interferometer was represented as a function of magnetic field. We follow the same strategy, by carefully analyzing first what happens to the transmittance at such avoided crossing points of the charging diagram. As we have mentioned, the two traces above regions $D$ and $C$ from Fig. 3(b) correspond to two eigenvalues $E_{i}\left(V_{1}, V_{2}\right)(i=1,2)$ that depend weakly on $V_{2}$. Similarly, the traces that approach $A$ and $D$ are associated with $E_{j}\left(V_{1}, V_{2}\right)$. Looking at Eqs. (24) and (26) one can notice that as long as $V_{2}$ does not align $E_{j}\left(V_{1}, V_{2}\right)$ to the Fermi level, the only terms that produce peaks in the transmittance are the ones involving $G^{(i)}$, and this happens each time when $E_{i}\left(V_{1}, V_{2}\right) \approx E_{F}$. The main point is that by varying $V_{2}$ we achieve the resonant condition for the term involving $G^{(j)}$, hence both dots will transmit. 

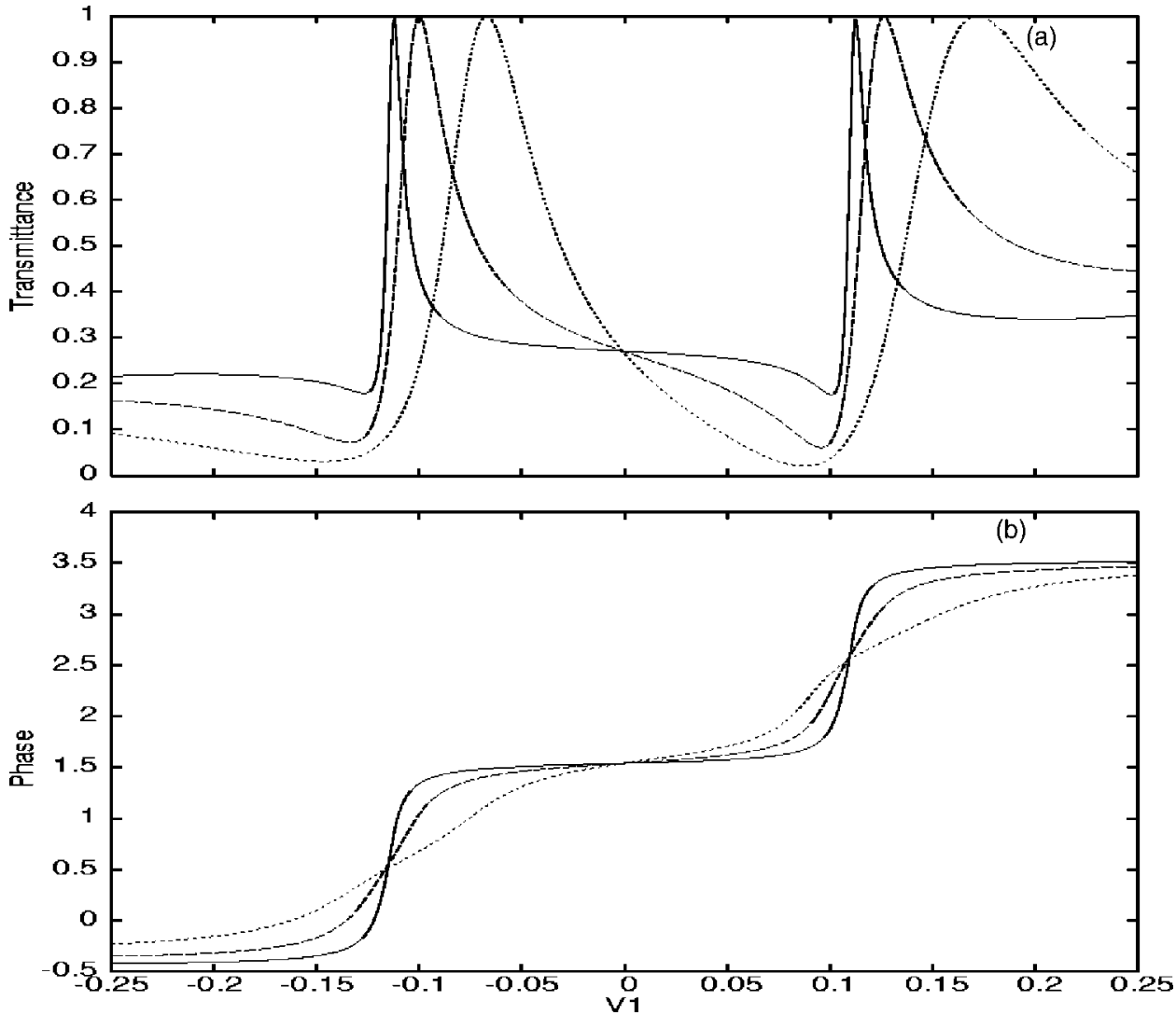

FIG. 9. The sharpness of the Fano resonances (a) and the phase of the transmittance through the interferometer (b), as a function of the interdot coupling, full line, $\tau_{\text {int }}=0.05$; dashed line, $\tau_{\text {int }}=0.15$; dotted line, $\tau_{\text {int }}=0.3$. At weak coupling the phase increases rapidly by $2 \pi$ while for stronger coupling it increases smoothly by $2 \pi$ along a Fano resonance. The parameters used are $V_{2}=-0.110, \phi=3, \tau$ $=0.3, \tau_{L}=1$.
In Fig. 5 we show a detail from the charging diagram in Fig. 3(b), taken in the neighborhood of almost crossing points $A$ and $B$. In contrast to the usual picture with sharp peaks here we observe [Fig. 5(a)] an asymmetric large tail of the peaks, which shows that in this regime the interferometer acts as a Fano system. This happens because one dot $\left(\mathrm{QD}_{2}\right)$ is always set to a resonance thus the corresponding arm of the ring is free, providing the continuum component for the interference. Formally this is easily understood by looking at Eq. (27), because the second term is always large enough and interfere with a quantity (the first term) that increases as $E_{i}(V)$ approaches the Fermi level. The Fano regime disappears quickly as we tune $\mathrm{QD}_{2}$ away from resonance, the picture of separate peaks being recovered [Fig. 5(b)].

In Figs. 6(a)-6(d) the solid lines are plots of the transmittance as a function of $V_{1}$ when $V_{2}$ is set close to a resonant value. Remark the sudden drop of the peak after the resonant point and the Fano dips. The latter are actually located in the avoided crossing region, which explains the small transmittance there. Moreover, the asymmetric tail changes its orientation as $V_{2}$ is slightly varied, i.e., the Fano parameter sign changes. Following Kobayashi et al. ${ }^{7}$ we shall call this feature the electrostatic control of the Fano asymmetric line. In order to explain this observation we must look at the two paths that are involved in the interference. The first contribution comes from the resonant tunneling through the upper dot and is given as dashed lines in Figs. 6(a)-6(d) (the plots were obtained by decoupling the lower arm of the ring from the leads). In this case there is no interference and one gets the usual resonant peaks. The second contribution is due to the background transmittance of the lower arm when $V_{2}$ is set close to a resonance and the upper arm does not transmit. We illustrate this component of transport in Fig. 6(e) which shows a single peak that appears by varying $V_{2}$ when $V_{1}$ is far away from resonant values. The points $A, B, C, D$ mark the magnitude of the background for four values of $V_{2}$. Clearly, as $V_{1}$ approaches the resonant points the interference becomes possible and the Fano lines appear. By inspecting each of Figs. 6(a)-6(d) in connection with Fig. 6(e) one gets a description of the line shape for different pairs of $V_{1}, V_{2}$. As long as the transmittance values of the two contributions are located on the same side of their corresponding peaks the interference is constructive and the Fano line increase up to a maximum which coincides with the resonant peak of the upper arm. In contrast, when $V_{1}, V_{2}$ are chosen such that the transmittance values are located on different sides of the peaks the two path interfere destructively and the Fano line drops to a dip. In particular, for $V_{2}$ fixed the dips will be located on the same side of the peaks, thus the Fano parameter conserve its sign. The appearance of Fano effect in interferometers with embedded dots was also discussed in a simple (exactly solvable) model in Ref. 32, without considering the interdot coupling or emphasizing the electrostatic control of the Fano line shape.

In the above discussion the magnetic flux was fixed and we have varied $V_{2}$, emphasizing the sensitivity of the Fano interference on this parameter. Figure 7 shows that the shape of the Fano line can be equally controlled by varying the magnetic flux, while keeping $V_{2}$ fixed. Indeed, as $\phi$ increases from 3.00 to 4.50 the asymmetric tail changes its orientation. This effect originates in the field dependence of the dot levels which leads in turn to a shift of the background peak. Indeed from Fig. 8(a) one notices at once that the back- 

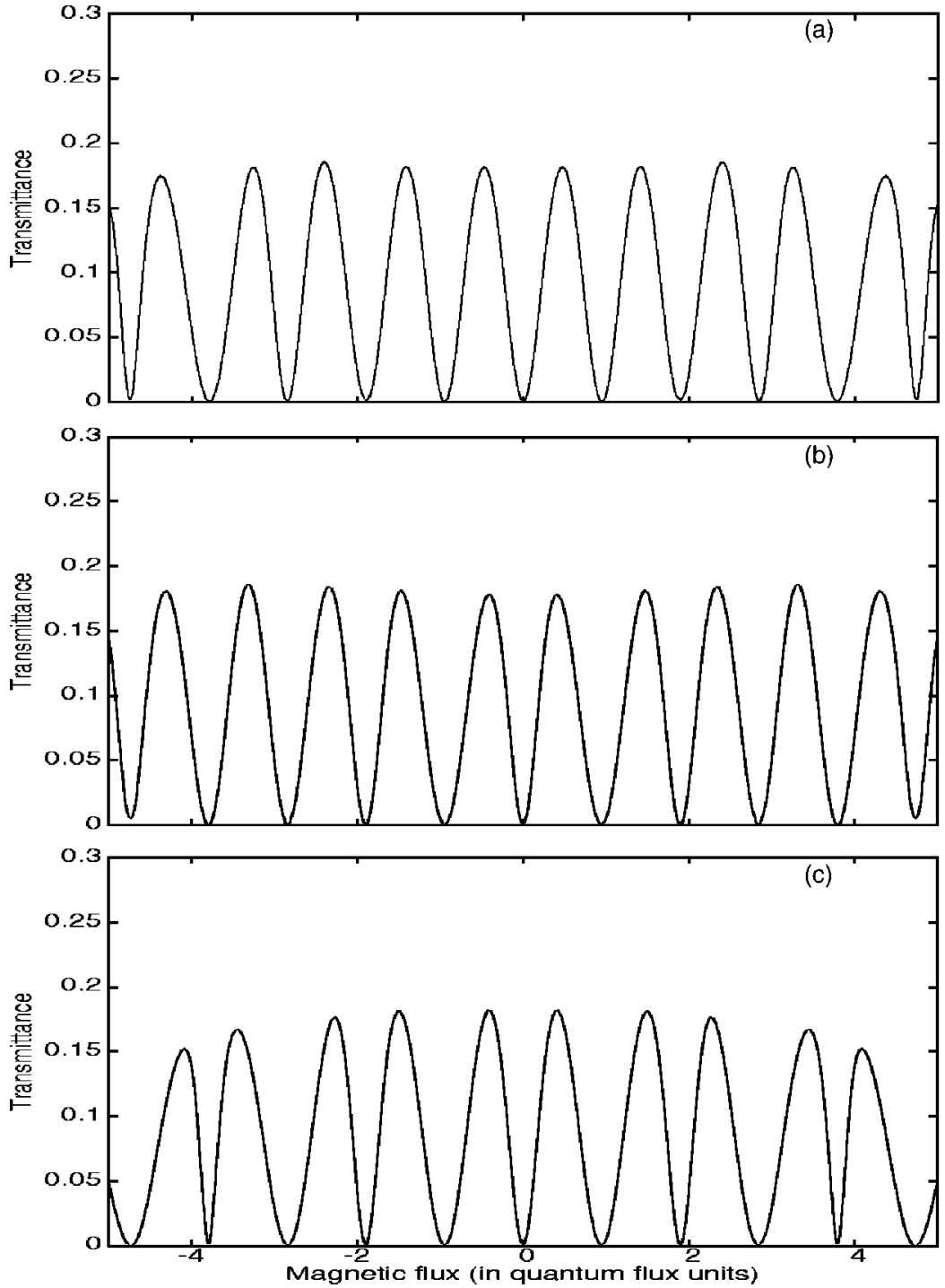

FIG. 10. The in-phase Aharonov-Bohm oscillations of the transmittance assigned to the Fano dips from the region $A, B, C$, and $D$ in Fig. 3(b). ground peak moves to the left as the magnetic flux is varied. In order to make the connection with Fig. 7 we marked with points the transmittance values corresponding to the gate voltage $V_{2}=0.11$. As a consequence of the magnetic shift the point located at $\phi=3.00$ on the left-hand side of the peak passed on the upper right-hand side at $\phi=3.80$ from where it goes down for $\phi=4.50$. The same argument used in the discussion of Fig. 6 explains now the change of the Fano parameter shown in Fig. 7.

Figure $8(\mathrm{~b})$ shows the $\phi$ - dependence of the resonant eigenvalue of the isolated double dot (the line obtained for a vanishing ring-dot coupling, i.e., $\tau=0)$ and of the eigenvalue of the whole interferometer (drawn at $\tau=0.3$ ). The horizontal lines mark the flux values chosen in Fig. 8(a). As expected a nonvanishing $\tau$ leads to a hybridization between the spectra of the truncated ring $\sigma\left(H^{R}\right)$ and the coupled dots $\sigma\left(H^{D}\right)$. The double-dot eigenvalue acquires a quasiperiodic modulation with $\phi$ due to the ring geometry.

By comparing Figs. 8(a) and 8(b) we observe that the background peak follows the field dependence of the eigenvalue of the isolated double dot and not the one of the interferometer eigenvalue. The physical meaning of this behavior is that the resonant transport is controlled by the spectral properties of the embedded dots. If the interferometer eigenvalue would control the peak position this one should move to the right from $\phi=3.00$ to $\phi=3.80$, according to the trajectory given for $\tau=0.3$. Clearly this is not the case and, up to a shift caused by the real part of the resonance the peak obeys the drift of the isolated eigenvalue. We stress that this nontrivial effect described above cannot be captured by a theoretical model that neglects the spectral properties of the dot in the magnetic field. The direction change of the asymmetric Fano tail at the variation of magnetic field was experimentally reported by Kobayashi et al. ${ }^{7}$ in the the case of a onedot interferometer. We believe that the effect we just discussed for the two-dots interferometer is similar.

We further investigate the behavior of the Fano peaks as a function of the interdot coupling. Figure 9(a) shows that the line shape is very sensitive to this parameter. More interesting is the behavior of the interferometer phase along a Fano resonance plotted in Fig. 9(b). For weak coupling (and hence for sharp peaks) the phase shows a rapid increase by $2 \pi$. This feature has some connection with the experimental results obtained in a single dot interferometer by Kobayashi et $a l .{ }^{7}$ They reported an increase of $2 \pi$ for the phase of the AB 


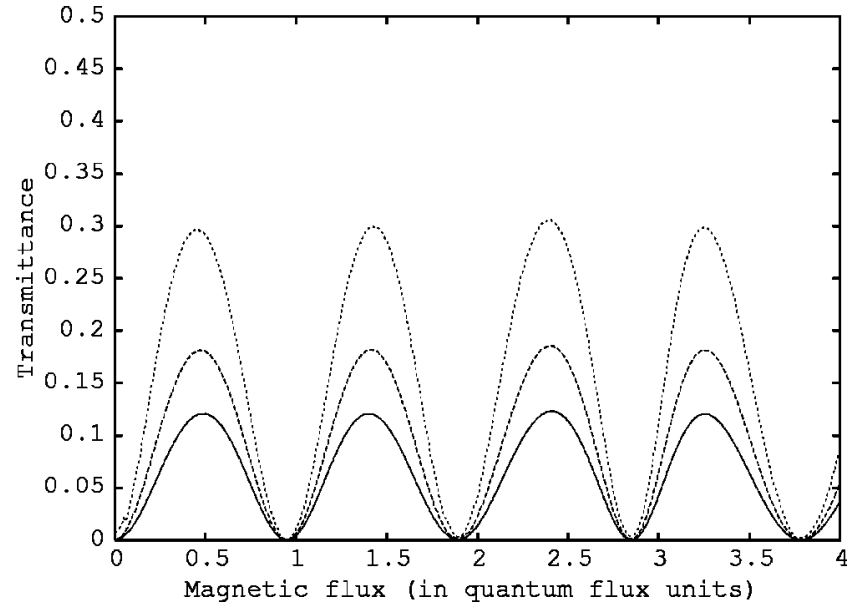

FIG. 11. Aharonov-Bohn oscillations in the region $D$ of the charging diagram at different interdot couplings, full line, $\tau_{\text {int }}=0.25$; dashed line, $\tau_{\text {int }}=0.2$; dotted line, $\tau_{\text {int }}=0.15$. Other parameters are $t_{L}=1, \tau=0.3, E_{F}=0$.

oscillations (we present instead the phase of the transmittance). In our case the second dot is set to a resonance so it acts as a free arm of the ring, from where the similarity with the one-dot interferometer. By increasing $\tau_{\text {int }}$ the phase becomes a smooth function of $V_{1}$.

We now address the problem of $\mathrm{AB}$ oscillations. It is clear that they are to be observed if both dots are close to resonance, meaning that the gate voltages $V_{1}, V_{2}$ are suitably tuned near some eigenvalues of the double dot. The delicate point is that the eigenvalues depend on the magnetic flux through the ring so that for different fluxes one needs different resonant values for $V_{1}, V_{2}$. Otherwise stated, the rhomboids move with $\varphi$ (not shown). We found that for small magnetic fields the changes are not too drastic and that the $\mathrm{AB}$ oscillations can be captured by monitoring the Fano dip and plotting the transmittance magnitude there as a function of the magnetic flux. More precisely, for a given magnetic flux we keep $V_{2}$ fixed and vary $V_{1}$ in a range that contains only one Fano dip whose transmittance is determined (this is simply the lowest value in the chosen range). Then we repeat the procedure for other fluxes, the results being given in Fig. 10. One can recognize at once the Aharonov-Bohm oscillations. Their position is slightly shifted due to the phase accumulation within the dots (i.e., we express the transmittance as a function of the magnetic flux through the ring while the flux encircled by the real trajectories is a bit larger). Notice that the oscillations are in phase at all Fano dips. Figure 11 shows that the oscillation amplitude increases as the interdot coupling decreases.

We have also investigated a single-dot interferometer (the ring has the same dimension while the dot is a $8 \times 9$ plaquette). When the free arm is decoupled (by making some hopping terms zero) we have the usual peaks corresponding to resonant tunneling via the dot levels [Fig. 12(a)]. In order to see the Fano features reported by Kobayashi et al. ${ }^{7}$ we restore the coupling to the arm and we choose the Fermi level such that the background conductance of the arm is around 0.3 (if the Fermi level coincides with some eigenvalue of the free arm its conductance approaches unity, obscuring thus the contribution of the dot). As expected, the symmetric peaks are turned to Fano resonances shown in Fig. 12(b), their correspondence being obvious. One notices that the Fano peaks are either wide or very narrow. We have checked that this feature remains also valid for other values of the flux and different number of sites composing the dot. Remarkably, the Fano parameter takes the same sign between succesive peaks. It was suggested recently by Nakan-
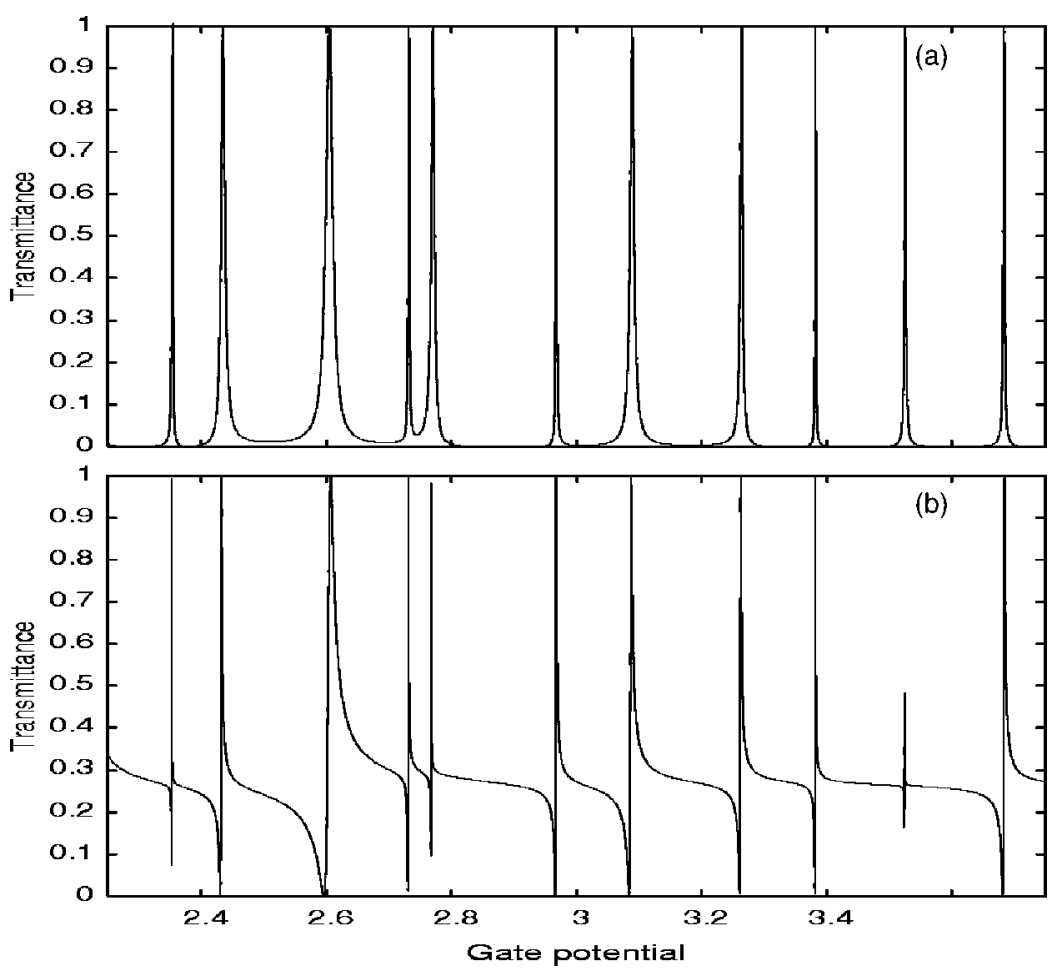

FIG. 12. Transmittance through a single-dot interferometer $\left(\tau_{L}=1, \tau=0.35, \phi=5, E_{F}=-0.5\right)$. The dot has $9 \times 8$ sites and the ring contains 100 sites. (a) Usual peaks arising from the resonant tunneling via the discrete levels of the dot (the free arm of the ring is decoupled). (b) The Fano regime, the free arm conducts and interferes with the path along the QD. The peaks turn to Fano line shapes. 
ishi et al. ${ }^{33}$ that this feature relates to the correlations between the narow and wide peaks.

\section{CONCLUSIONS}

The main aim of this paper was to present in a unified formalism the basic properties of Aharonov-Bohm interferometers with coupled quantum dots. By combining the Landauer-Büttiker approach and the Feschbach formula we studied the transport properties of the interferometer in terms of the spectral properties of the embedded dots. Our method involves only Green functions and can be viewed as an alternative to the scattering theoretical approach. In the case of an interferometer with two coupled QD (one QD in each arm of the ring) we give a formula [Eq. (27)] which emphasizes the resonant tunneling process through a given discrete level from the dots (we recall that along the paper we have considered many-level dots).

Numerical simulations reproduce the stability charging diagrams of two-dot $\mathrm{AB}$ interferometer reported in the experiments of Holleitner et al. ${ }^{5}$ A careful analysis of the almost crossing points of the diagram lead us to several interesting results which are summarized in what follows. When the magnetic field is fixed and one dot is set to resonance the interferometer transmittance shows Fano line shapes as a function of the gate voltage applied to the other dot. This corroborates with the results of Kubala and König ${ }^{32}$ obtained in an exactly solvable one-site model and shows clearly the coherent feature of the transport through the system. We emphasized and explained the sensitivity of the Fano tail to the gate potential on the second dot.

As we have said, our model includes the effect of the magnetic field on the dot levels. It turned out that this effect explains the change of the asymmetric tail as the magnetic flux is varied. It would be of great interest to probe experimentally this latter aspect. The transmittance assigned to the Fano dips shows Aharonov-Bohm oscillations, in full agreement with the observations of Holleitner et al. ${ }^{5}$ The influence of the various coupling constants was identified. Finally we reproduced the results of Kobayashi et al. ${ }^{7}$

The analysis of the 4-lead geometry in view of the very recent results reported in the study by Sigrist et al. ${ }^{6}$ is much more complex and requires further investigation.

\section{ACKNOWLEDGMENTS}

This work was supported by Grant CNCSIS and Romanian Programme for Fundamental Research. V.M. acknowledges support from the NATO-TUBITAK and the Romanian Ministry of Education and Research under CERES contract. B.T. acknowledges the support of TUBITAK, NATO-SfP, MSB-KOBRA, and TUBA. The authors are very grateful to Ulrich Wulf for many valuable discussions.
${ }^{1}$ A. Yacoby, M. Heiblum, D. Mahalu, and Hadas Shtrikman, Phys. Rev. Lett. 74, 4047 (1995).

${ }^{2}$ A. L. Yeyati and M. Büttiker, Phys. Rev. B 52, R14360 (1995).

${ }^{3}$ R. Schuster, E. Bucks, M. Heiblum, D. Mahalu, V. Umanski, and Hadas Shtrikman, Nature (London) 385, 417 (1997).

${ }^{4}$ E. Bucks, R. Schuster, M. Heiblum, D. Mahalu, and V. Umanski, Nature (London) 391, 871 (1998).

${ }^{5}$ A. W. Holleitner, C. R. Decker, H. Qin, K. Eberl, and R. H. Blick, Phys. Rev. Lett. 87, 256802 (2001); A. W. Holleitner, H. Qin, K. Eberl, and J. P. Kotthaus, Physica E (Amsterdam) 12, 774 (2002).

${ }^{6}$ M. Sigrist, et al., Phys. Rev. Lett. 93, 066802 (2004).

${ }^{7}$ Kensuke Kobayashi, Hisashi Aikawa, Shingo Katsumoto, and Yasuhiro Iye, Phys. Rev. Lett. 88, 256806 (2002); Phys. Rev. B 68, 235304 (2003).

${ }^{8}$ U. Fano, Phys. Rev. 124, 1866 (1961).

${ }^{9}$ J. U. Nockel, and A. D. Stone, Phys. Rev. B 50, 17415 (1994).

${ }^{10}$ E. R. Racec and U. Wulf, Phys. Rev. B 64, 115318 (2001).

${ }^{11} \mathrm{G}$. Hackenbroich and H. A. Weidenmüller, Phys. Rev. Lett. 76, 110 (1996).

${ }^{12}$ G. Hackenbroich and H. A. Weidenmüller, Phys. Rev. B 53, 16379 (1996)

${ }^{13}$ G. Hackenbroich, Phys. Rep. 343, 463 (2001).

${ }^{14}$ J. König and Y. Gefen, Phys. Rev. B 65, 045316 (2002).

${ }^{15}$ B. Kubala and J. König, Phys. Rev. B 67, 205303 (2003).

${ }^{16}$ H. A. Weidenmüller, Phys. Rev. B 65, 245322 (2002).

${ }^{17}$ O. Entin-Wohlman, A. Aharony, Y. Imry, Y. Levinson, and A. Schiller, Phys. Rev. Lett. 88, 166801 (2002).

${ }^{18}$ A. Aharony, O. Entin-Wohlman, B. I. Halperin, and Y. Imry,
Phys. Rev. B 66, 115311 (2002).

${ }^{19}$ A. Aharony, O. Entin-Wohlman, and Y. Imry, Turk. J. Phys. 27, 299 (2003).

${ }^{20}$ H. Feschbach, Ann. Phys. (N.Y.) 5, 363 (1958).

${ }^{21}$ G. Nenciu, Rev. Mod. Phys. 63, 91 (1991).

${ }^{22}$ V. Moldoveanu, A. Aldea, A. Manolescu, and M. Nita, Phys. Rev. B 63, 045301 (2001).

${ }^{23}$ A. Aldea, V. Moldoveanu, M. Nita, A. Manolescu, V. Gudmundsson, and B. Tanatar, Phys. Rev. B 67, 035324 (2003).

${ }^{24}$ V. Moldoveanu, A. Aldea, and B. Tanatar, Phys. Rev. B 70, 085303 (2004)

${ }^{25}$ H. D. Cornean, A. Jensen, and V. Moldoveanu, Report No. mparc 04-71, available at http://www.ma.utexas.edu

${ }^{26}$ S. Datta, Electronic Transport in Mesoscopic Systems (Cambridge University Press, Cambridge, 1995).

${ }^{27}$ F. Ramirez, E. Cota, and S. E. Ulloa, Phys. Rev. B 59, 5717 (1999).

${ }^{28}$ R. Ziegler, C. Bruder, and H. Schoeller, Phys. Rev. B 62, 1961 (2000).

${ }^{29}$ W. G. van der Wiel, S. De Franceschi, J. M. Elzerman, T. Fujisawa, S. Tarucha, and L. P. Kouwenhoven, Rev. Mod. Phys. 75, 1 (2003).

${ }^{30}$ F. R. Waugh, M. J. Berry, D. J. Mar, R. M. Westervelt, K. L. Campman, and A. C. Gossard, Phys. Rev. Lett. 75, 705 (1995).

${ }^{31}$ H. A. Weidenmüller, Phys. Rev. B 68, 125326 (2003).

${ }^{32}$ B. Kubala and J. König, Phys. Rev. B 65, 245301 (2002).

${ }^{33}$ T. Nakanishi, K. Terakura, and T. Ando, Phys. Rev. B 69, 115307 (2004). 